\title{
PAPER
}

\section{Deep brain stimulation of the subthalamic nucleus: anatomical, neurophysiological, and outcome correlations with the effects of stimulation}

\author{
M M Lanotte, M Rizzone, B Bergamasco, G Faccani, A Melcarne, L Lopiano
}

See end of article for authors' affiliations

Correspondence to

Dr M M Lanotte, Corso

Stati Uniti 53, 10129

Torino, Italy;

m.lanotte@tin.it

Received

29 December 2000

In revised form 15 June

2001

Accepted 26 June 2001

\begin{abstract}
Objectives: Bilateral chronic high frequency stimulation of the subthalamic nucleus (STN), through the stereotactical placement of stimulating electrodes, effectively improves the motor symptoms of severe Parkinson's disease. Intraoperative neurophysiological and clinical monitoring techniques (neuronal electrical activity recording and intraoperative stimulation) may improve and refine the localisation of the nucleus. The objective of this work was to compare the preoperative CT and MRI localisation with the intraoperative neurophysiological identification of STN. The relation between the localisation of the STN and the position of the most effective contact of the permanent quadripolar electrode at a 3 month and 1 year follow up was also studied.

Methods: Fourteen consecutive parkinsonian patients were submitted to bilateral implant for STN stimulation. All the patients underwent a standard MRI and stereotactic CT to obtain, by image fusion and localisation software, the stereotactical coordinates of STN. The STN extension and boundaries were identified by a semimicrorecording of the neuronal electrical activity. The definitive quadripolar electrode was positioned to locate at least two contacts within the STN recording area. Intraoperative macrostimulation was performed to confirm the correct position of the electrode. Postoperative clinical evaluation of the effects of stimulation was checked for each contact of the quadripolar electrode testing the improvement on contralateral rigidity to select the best contact. This evaluation was repeated at 3 months and 1 year after surgery.

Results: In $35.7 \%$ of the procedures it was necessary to perform more than one track to get a recording of neuronal activity consistent with STN.

The mean position of the central point of all the 28 STN recording areas in respect of the AC-PC line midpoint was $2.7 \mathrm{~mm}$ posterior (SD 0.7), $3.8 \mathrm{~mm}$ inferior (SD 1.1), and $11.6 \mathrm{~mm}$ lateral (SD 0.9), and the mean distance between the anatomical target and the central point of the STN as defined by intraoperative recording was $0.5 \mathrm{~mm}$ (SD 0.5) on the anteroposterior plane, $0.7 \mathrm{~mm}$ (SD 0.7) on the lateral plane, and $0.9 \mathrm{~mm}$ (SD 0.6) on the vertical plane. At 1 year the mean position of the central point of the most effective contact of the electrode in respect of the AC-PC line midpoint was $1.7 \mathrm{~mm}$ posterior (SD 0.9), $1.7 \mathrm{~mm}$ inferior (SD 1.5), and $12.3 \mathrm{~mm}$ lateral (SD 0.9).

Conclusion: The results highlight the role of the intraoperative recording to get a more accurate localisation of the STN in surgery for Parkinson's disease, allowing the identification of the boundaries and of the extension of the nucleus. The most effective contact of the quadripolar electrode was always in the upper part of the STN recording area or immediately above it, suggesting a role of this region in the clinical effectiveness of the STN electrical stimulation.
\end{abstract}

$\mathrm{D}$ eep brain stimulation (DBS) has an increasing role in the treatment of movement disorders, mainly Parkinson's disease. ${ }^{1-3}$ Different anatomical targets have been considered for Parkinson's disease: the ventralis intermedius nucleus (VIM), subthalamic nucleus (STN), and internal pallidum (GPi). ${ }^{4-8}$ It has been reported that STN stimulation effectively improves motor symptoms of severe Parkinson's disease through a functional inhibition of the subthalamic neurons. ${ }^{9-12}$ Many reports have shown a remarkable improvement in off period duration and in the activities of daily living score ${ }^{13-16}$; moreover, the clinical improvement allowed the reduction of dopaminergic drugs resulting in a significant decrease of dyskinesias. A preliminary report showed long term improvement of parkinsonian symptoms-up to 3 years. ${ }^{17}$

Different methods exist for anatomical localisation of the target; the STN may be directly visualised by T2 weighted coronal MRI sections, even though this technique may be affected by the typical MRI distorsion that influences stereotactic precision. ${ }^{18}{ }^{19}$ The STN may also be localised by an indirect procedure following a stereotactic atlas, calculating its position through the distance from the anterior commissure (AC)-posterior commissure (PC) line. The keystone of this system is the correct identification of both AC and PC. For this purpose ventriculography may be used and, probably, it is the most reliable procedure for the anatomical targeting of STN. ${ }^{40}$ A recent choice is represented by image fusion of standard MRI sections and CT, taking the better anatomical detail of the first and the better geometric precision of the second. ${ }^{21}$

Another problem may arise from individual variability with respect to the atlas; this may result in an incorrect implantation of the electrodes. Intraoperative neurophysiological and

Abbreviations: DBS, deep brain stimulation; VIM, ventralis intermedius nucleus; STN, subthalamic nucleus; GPi, internal pallidum; AC, anterior commissure; PC, posterior commissure; UPDRS, unified Parkinson's disease rating scale; IPG, implantable pulse generator; $M E c 0$, most effective contact; Aco, contact immediately above the MEco; Bco, contact immediately below the MEco 
clinical monitoring techniques (neuronal electrical activity recording and intraoperative stimulation) may improve and refine the anatomical localisation. ${ }^{13} 1822$ With micro or semimicrorecording, when the electrode reaches the target a typical change in electrical activity occurs, due to the sustained pattern of discharge of STN neurons (firing rate $25 \mathrm{~Hz}-45$ $\mathrm{Hz}){ }^{22}$ Additionally, the neurons can be activated by specific contralateral proprioceptive stimuli or they can show an intrinsic rhythmic discharge synchronous with the parkinsonian tremor. ${ }^{22}{ }^{23}$ Therefore, recording procedures can allow the identification of the STN boundaries, and intraoperative stimulation provides an evaluation of the clinical efficacy on contralateral rigidity, akinesia, and tremor and a check of the adverse side effect threshold, adding important information about the optimal targeting. ${ }^{13}$

In this study we tested the reliability of our anatomical targeting system, based on the fusion between MRI sections and $\mathrm{CT}$, stressing the role of the intraoperative neurophysiological and clinical findings in reaching an optimal electrode position within the STN. The aim of the study was to compare the anatomical localisation with the neurophysiological identification of the STN. We also report the correlation between the position of the most effective contact of the definitive quadripolar electrode and the anatomical-neurophysiological targeting of the STN.

\section{METHODS \\ Patients}

Fourteen consecutive patients with Parkinson's disease (nine men and five women) were submitted to bilateral implant for STN stimulation between October 1998 and November 1999. Their ages ranged from 49 to 70 years, with a mean of 60.4 (SD 6.7) years. Mean duration of disease was 14.9 (SD 5.5) years and mean duration of levodopa treatment was 15.3 (SD 5.0) years. Preoperative average Hoehn and Yahr stage was 2.4 (SD 0.3 ) in the medication on (med on) condition and 4.3 (SD 0.4) in the medication off (med off) condition. The unified Parkinson's disease rating scale (UPDRS) ${ }^{24}$ part II average score was 8.7 (SD 6.9) in the med on condition and 27.7 (SD 4.6) in the med off condition. The UPDRS part III average score was 20.4 (SD 8.9) in the med on condition and 59.1 (SD 14.6) in the med off condition. All the patients showed significant on/off daily fluctuations and the on state was affected by drug related involuntary movements with resulting disability (UPDRS part IV average score 10.5) (SD 2.7).

All the patients gave their written consent to the study.

\section{Surgical procedure}

All the patients were submitted to a standard MRI (SPGR sequences with $2 \mathrm{~mm}$ thick contiguous slices). At surgery a Cosman-Roberts-Wells stereotactic frame (CRW, Radionics, Inc, Burlington, MA, USA) was placed, trying to be parallel to the AC-PC line by visually aligning the anteroposterior axis with the imaginary line connecting the external auditory canal with the inferior orbital wall. Special attention was paid to prevent significant tilt or rotation of the frame with respect to the brain. Brain CT was then performed with $2 \mathrm{~mm}$ thick contiguous slices. Subsequently both MRI and stereotactic CT images were loaded on Stereoplan apparatus (Radionics, Inc, Burlington, MA, USA) and fused by the Image Fusion software to combine the advantage of CT spatial accuracy with the superior tissue definition of MRI. Image Fusion software employs algorithms that allow a bone match or a voxel by voxel intensity match.

Both AC and PC were detected by Stereoplan software and the STN target was defined as $2.5 \mathrm{~mm}$ posterior and $4 \mathrm{~mm}$ inferior to the midcommissural point, and $12 \mathrm{~mm}$ from the midline. It was therefore chosen as the best track to the target, using a $58^{\circ}-63^{\circ}$ anteroposterior angle and a $14^{\circ}-20^{\circ}$ lateral angle; three dimensional trajectory visualisation allowed the avoidance of critical structures, such as the ventricles or the motor strip. Atlas plan software based on the SchaltenbrandWahren "atlas for stereotaxy of the human brain ${ }^{25}$ provided an aid in confirming target and trajectory.

With local anaesthesia a $14 \mathrm{~mm}$ precoronal burr hole was made. A semimicroelectrode (SME, Radionics Inc, Burlington, MA, USA) with a recording surface of $50 \mu \mathrm{m}^{2}$ and $0.5-1 \mathrm{M} \Omega$ impedance, was progressively advanced along the trajectory by a digital micromanipulator (CRW-PMD, Radionics Inc, Burlington, MA, USA) and the electrical cell activity was recorded starting from $7 \mathrm{~mm}$ above the target. The signal was amplified, filtered, and displayed during intraoperative recording by Neuromap system (Radionics Inc, Burlington, MA, USA).

The STN was identified by the following criteria: change in the background noise due to the recording of high amplitude spikes with a firing rate of $25-45 \mathrm{~Hz}$; the presence of "tremor cells" with an oscillatory discharge at the same frequency as the tremor $(4 \mathrm{~Hz}-6 \mathrm{~Hz})$; identification of cells responsive to passive contralateral movements. ${ }^{22}$ When the intraoperative recording did not show typical STN electrical activity, we performed a macrostimulation using a train of impulses of 130 $\mathrm{Hz}, 60 \mu \mathrm{s}$, and a voltage from 0.5 to $6 \mathrm{~V}$ to highlight the occurrence of side effects. On the basis of the characteristics of these side effects another track was made, moving posteromedially when muscle contractions occurred and laterally when paraesthesias appeared. A DBS 3389 chronic stimulation electrode with four contacts (Medtronic Inc, Minneapolis, MN, USA) was finally inserted to locate at least two contacts in the STN recording area. Monopolar cathodic stimulation was then performed with each contact to evaluate the clinical effect and the adverse side effect threshold (Test Stimulator Kit, mod 3625, Medtronic Inc, Minneapolis, MN, USA). After a fluoroscopic control to verify that there was no gross modification of its position, the electrode was secured to the skull and connected to its temporary percutaneous extension. The day after surgery patients underwent MRI to exclude any surgical complications.

A few days after the electrode implantation, general anaesthesia was induced and an implantable pulse generator (IPG) (Medtronic Itrel II, Medtronic Inc, Minneapolis, MN, USA) was bilaterally located in a subcutaneous pocket in the infraclavicular region; it was finally connected to the distal tip of the electrode by an extension cable passed subcutaneously up the neck to the parietal region.

\section{Electrical stimulation setting}

A week after surgery, the clinical evaluation of the effects of stimulation was performed for each contact of the definitive quadripolar electrode, analysing the improvement on contralateral rigidity (UPDRS part III, item 22) and the side effect voltage threshold, after a 12 hour withdrawal of all antiparkinsonian drugs. Only one pulse generator was switched on at a time (to avoid any possible homolateral effect of stimulation) and the effect of stimulation was evaluated on the different contacts with progressively higher voltage $(0.2 \mathrm{~V}$ increments, from 0 to $6-7 \mathrm{~V}$ ) with a rate of $130 \mathrm{~Hz}$ and a pulse width of $60 \mu \mathrm{s}$, using a monopolar cathodic stimulation. During the clinical evaluation, both the patient and the examiner were unaware of the state of stimulation. At first we identified the contact capable of determining the best clinical effect; stimulating through this contact, we found the minimum voltage capable of obtaining the best improvement on contralateral wrist rigidity. Finally we compared the effect of the stimulation with this voltage on the different contacts.

The contacts identified as the most effective (lower voltage to obtain the clinical effect; higher side effect threshold) were therefore used for chronic stimulation. During the early postoperative period the voltage of stimulation was progressively increased, decreasing the levodopa dosage at the same time.

At 3 months and 1 year the whole procedure was repeated to check the efficacy of the different contacts of the electrodes. 


\section{Clinical evaluation}

The clinical condition of the patients after the surgery was assessed by the unified Parkinson's disease rating scale ${ }^{24}$ at 3 months and 1 year.

The clinical evaluation was performed in the morning, after a 12 hour withdrawal of antiparkinsonian drugs, studying the patient in the stimulation (stim) off/med off and in the stim on/med off conditions, to highlight the clinical effect of the stimulation alone. The stimulation parameters at the moment of the study were the same as those used during chronic stimulation, and the patients, but not the examiner, were unaware of the stimulation condition.

\section{Statistical analysis}

Statistical analysis of the data was performed by Wilcoxon signed rank test and by Kruskal-Wallis test for the nonparametric data and by analysis of variance (ANOVA) for parametric data. To indicate statistical significance a $\mathrm{p}$ value $<0.05$ was required.

\section{RESULTS}

Fourteen patients were bilaterally implanted in the STN. However, it was necessary to perform a total number of 38 tracks: in seven cases $(25 \%)$, on the basis of the recording pattern and
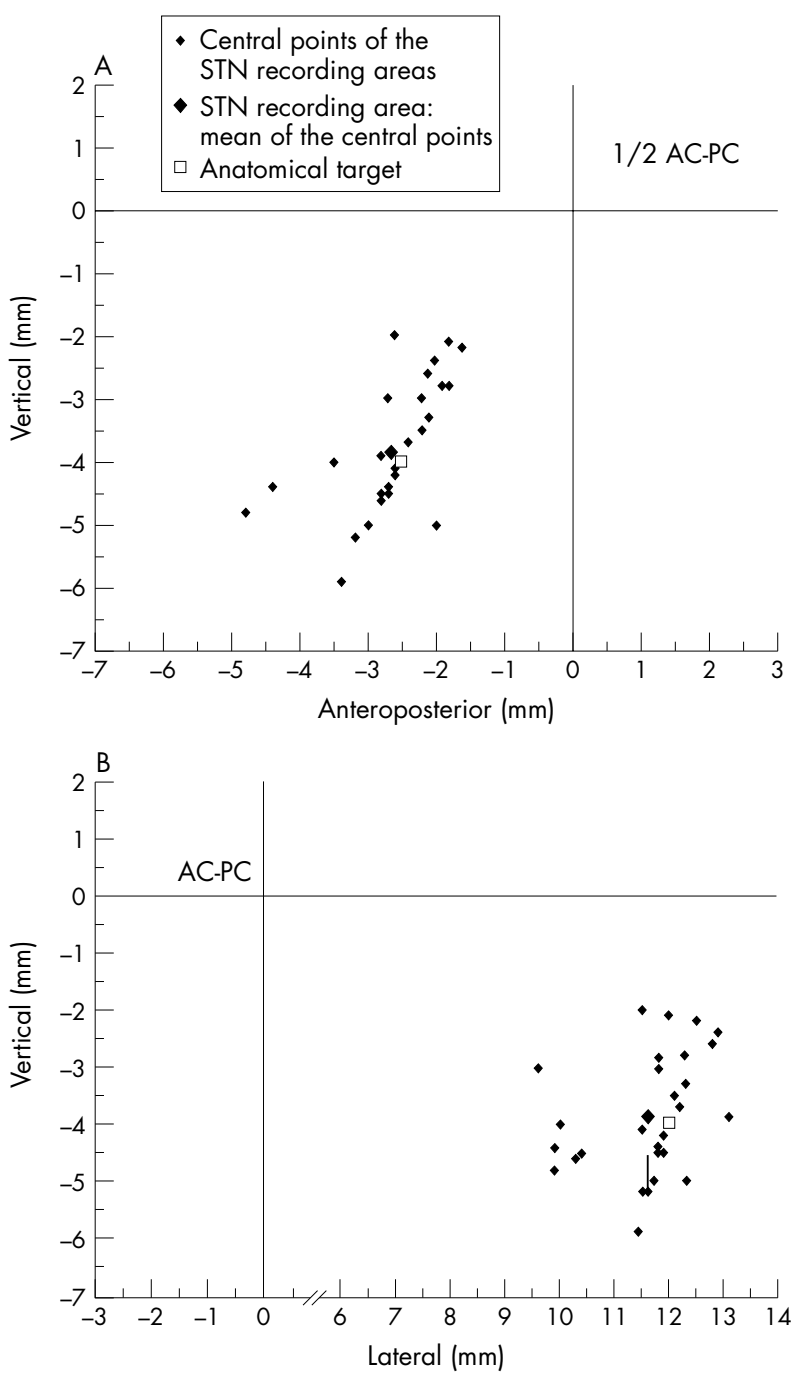

Figure 1 (A) Position of the anatomical target and of the central points of the subthalamic nucleus as determined by semimicrorecording (sagittal plane). (B) Position of the anatomical target and of the central points of the subthalamic nucleus as determined by semimicrorecording (coronal plane). the effects of the intraoperative macrostimulation, both indicative of an incorrect targeting of STN, we performed a second trajectory; in three of these cases $(10.7 \%$ of the total number of procedures) a third track was necessary.

The mean corrections of the calculated stereotactical coordinates necessary to reach the target in the 10 adjunctive tracks were $0.8 \mathrm{~mm}$ (SD 0.6) posterior on the sagittal plane and $1.4 \mathrm{~mm}$ (SD 1.2) medial on the coronal plane; it was not necessary to make any correction on the vertical plane.

The changes of cellular electrical activity when the semimicroelectrode reached the STN allowed the identification of the upper and lower boundaries of the nucleus and the calculation of the midpoint of the recording area with respect to the AC-PC line. The average length of the AC-PC line in our patients was $24.5 \mathrm{~mm}$ (SD 1.1).

The average length of the STN recording areas was $4.2 \mathrm{~mm}$ (SD 0.9). The mean position of the central point of the 28 STN recording areas in respect of the AC-PC line midpoint was 2.7 $\mathrm{mm}$ posterior (SD 0.7), $3.8 \mathrm{~mm}$ inferior (SD 1.1), and $11.6 \mathrm{~mm}$ lateral (SD 0.9) (fig $1 \mathrm{~A}$ and B). The average distance between the location of the centre of the STN as determined by semimicrorecording and that of the STN as determined by the stereotactical coordinates was $0.5 \mathrm{~mm}$ (SD 0.5) on the anteroposterior plane, $0.7 \mathrm{~mm}$ (SD 0.7) on the lateral plane, and 0.9 mm (SD 0.6) on the vertical plane.

As described, we tested the clinical efficacy of the stimulation on the different contacts of the electrodes 3 months and l year after the surgery, scoring the effect on contralateral wrist rigidity. The average baseline rigidity score at the wrist (stim off/med off condition) was 2.5 (SD 0.7) at 3 months and 2.4 (SD 0.7) at 1 year. No significant differences were found between the two conditions ( $W=32.0 ; \mathrm{p}>0.050)$. The stimulation through the most effective contact (MEco) brought about a significant reduction of the average score to 0.3 (SD 0.3) at 3 months and to 0.2 (SD 0.3) at 1 year. Stimulating with the contact immediately above the MEco (Aco), the mean rigidity score improved to 1.2 (SD 0.7) at 3 months and to 0.9 (SD 0.6) at 1 year, whereas using the contact immediately below the MEco (BCo) the average score was 1.5 (SD 0.8) at 3 months and 1.1 (SD 0.7) at 1 year. The differences of the effects of the stimulation between the MEco and the Aco ( 3 months: $W=$ -288.0 ; $\mathrm{z}$ sub $W=4.169$; $\mathrm{p}=0.000$; 1 year: $W=-283.0$; $\mathrm{z}$ sub $W=3.860 ; \mathrm{p}=0.000)$ and between the MEco and the Bco ( 3 months: $W=-316.0 ; \mathrm{z}$ sub $W=4.275 ; \mathrm{p}=0.000 ; \mathrm{l}$ year: $W=-315.0 ; \mathrm{z}$ sub $W=4.275 ; \mathrm{p}=0.000$ ) were significant at 3 months and at 1 year. There were no significant differences between the Aco and the Bco ( 3 months: $W=76.0$; $\mathrm{z}$ sub $W=1.257 ; \mathrm{p}=0.209 ; \mathrm{l}$ year: $W=65.0 ; \mathrm{z}$ sub $W=1.172$; $\mathrm{p}=0.241$ ). Moreover, no significant differences were found comparing the effect of the stimulation through the same contact at 3 months and at 1 year (MEco 3 months $v$ MEco 1 year: $W=27.0 ; \mathrm{p}>0.050$; Aco 3 months $v$ Aco 1 year: $W=44.0$; $\mathrm{p}>0.050$; Bco 3 months $v$ Bco 1 year: $W=41.0 ; \mathrm{p}>0.050)$. The mean voltage of the stimulus necessary to obtain the best clinical effect on wrist rigidity was $2.7 \mathrm{~V}$ ( SD 0.5) at 3 months and $2.8 \mathrm{~V}$ (SD 0.6) at 1 year; no significant differences were found between these two values ( $W=111.0$; z sub $W=1.803$; $\mathrm{p}=0.071$ ).

The STN stimulation using MEcos gave a great improvement in the patients' clinical condition: at 3 months the average motor UPDRS (part III) score significantly improved from 56.5 (SD 18.2) in the stim off/med off condition to 26.4 (SD $9.8)$ in the stim on/med off condition $(W=91.0 ; \mathrm{p}<0.022)$; at 1 year follow up the average motor UPDRS score significantly improved from 62.3 (SD 13.4) in the stim off/med off condition to 24.1 (SD 9.3) in the stim on/med off condition $(W=78.0 ; \mathrm{p}<0.020)$. The difference between the UPDRS motor scores in the stim off/med off conditions at 3 months and 1 year was not significant $(W=-29.0 ; \mathrm{p}>0.052)$. The average UPDRS part II score was 10 (SD 6.3) at 3 months and 12.5 (SD 7.4 ) at 1 year, while the average UPDRS part IV score was 3 (SD 3.4) at 3 months and 2.8 (SD 2.2) at 1 year. 


\begin{tabular}{|c|c|c|c|c|c|}
\hline & \multicolumn{2}{|c|}{$\begin{array}{l}\text { Distance from the centre of the STN } \\
\text { recording area }\end{array}$} & \multicolumn{3}{|c|}{$\begin{array}{l}\text { Statistical comparison between } 3 \text { months } \\
\text { and } 1 \text { year data }\end{array}$} \\
\hline & 3 months & 1 year & $\mathrm{DF}$ & $F$ & $\mathrm{p}$ Value \\
\hline \multicolumn{6}{|l|}{ MEco: } \\
\hline$A P$ & $0.8(0.6)$ & $0.9(0.5)$ & 55 & 0.265 & 0.609 \\
\hline Lat & $0.6(0.5)$ & $0.7(0.4)$ & 55 & 0.308 & 0.581 \\
\hline Vert & $1.7(1.1)$ & $1.9(1.0)$ & 55 & 0.298 & 0.587 \\
\hline \multicolumn{6}{|l|}{ Aco: } \\
\hline AP & $1.6(0.6)$ & $1.8(0.6)$ & 51 & 0.560 & 0.458 \\
\hline Lat & $1.2(0.5)$ & $1.3(0.5)$ & 51 & 0.415 & 0.522 \\
\hline Vert & $3.5(1.1)$ & $3.7(0.9)$ & 51 & 0.615 & 0.437 \\
\hline \multicolumn{6}{|l|}{ Bco: } \\
\hline AP & $0.4(0.3)$ & $0.4(0.2)$ & 54 & 0.690 & 0.410 \\
\hline Lat & $0.4(0.3)$ & $0.3(0.3)$ & 54 & 0.309 & 0.580 \\
\hline \multirow[t]{3}{*}{ Vert } & $0.9(0.6)$ & $0.8(0.6)$ & 54 & 0.548 & 0.462 \\
\hline & \multicolumn{2}{|c|}{$\begin{array}{l}\text { Distance from the upper limit of the } \\
\text { STN recording area }\end{array}$} & \multicolumn{3}{|c|}{$\begin{array}{l}\text { Statistical comparison between } 3 \text { months } \\
\text { and } 1 \text { year data }\end{array}$} \\
\hline & 3 months & 1 year & $\mathrm{DF}$ & $F$ & $\mathrm{p}$ Value \\
\hline \multicolumn{6}{|l|}{ MEco: } \\
\hline AP & $0.5(0.3)$ & $0.4(0.3)$ & 55 & 0.975 & 0.328 \\
\hline Lat & $0.4(0.3)$ & $0.3(0.3)$ & 55 & 1.222 & 0.274 \\
\hline Vert & $1.0(0.7)$ & $0.9(0.7)$ & 55 & 0.584 & 0.448 \\
\hline \multicolumn{6}{|l|}{ Aco: } \\
\hline AP & $0.7(0.5)$ & $0.8(0.4)$ & 51 & 0.702 & 0.406 \\
\hline Lat & $0.5(0.4)$ & $0.6(0.4)$ & 51 & 0.519 & 0.474 \\
\hline Vert & $1.5(1.0)$ & $1.7(0.9)$ & 51 & 0.562 & 0.457 \\
\hline \multicolumn{6}{|l|}{ Bco: } \\
\hline AP & $1.0(0.6)$ & $0.9(0.5)$ & 54 & 0.285 & 0.595 \\
\hline Lat & $0.8(0.5)$ & $0.7(0.5)$ & 54 & 0.188 & 0.666 \\
\hline Vert & $2.1(1.0)$ & $1.9(0.9)$ & 54 & 0.301 & 0.585 \\
\hline
\end{tabular}

For each patient we calculated the position of the central point of each of the four contacts of the permanent electrode in respect of the midcommissural point, as determined by stereotactical coordinates, taking into account the angle of penetration of the electrode. At 3 months the mean position of the central point of the MEco in respect of the AC-PC line midpoint was $1.8 \mathrm{~mm}$ posterior (SD 1.0), $1.9 \mathrm{~mm}$ inferior (SD 1.7), and $12.2 \mathrm{~mm}$ lateral (SD 0.9). In three cases we found that the MEco at 1 year was different from the one identified at 3 months, despite the fact that the difference in clinical efficacy between the contacts was very slight $(0.5$ at the UPDRS item 22 score for the contralateral wrist rigidity). In all of these cases the MEcos at 1 year were in a higher position and therefore, considering the double obliquity of the electrode, more anterodorsolateral. The mean position of the central point of the MEco in respect of the AC-PC line midpoint changed therefore to $1.7 \mathrm{~mm}$ posterior (SD 0.9), 1.7 $\mathrm{mm}$ inferior (SD 1.5), and $12.3 \mathrm{~mm}$ lateral (SD 0.9).

For each patient we calculated at 3 months and at 1 year the distance between the central point and the upper limit of the STN recording area and the central point of the MEco, the Aco,and the Bco. Table 1 shows the mean values for these distances relative to the 28 electrodes. No significant differences were found for these distances between 3 months and 1 year. At 3 months one MEco was between the lower limit of the STN recording area and its centre, 15 MEcos were between the centre and the upper limit (inside $=$ MEco I 3 months), and 12 MEcos were above the upper limit (outside $=$ MEco O 3 months). At 1 year no MEcos were below the centre of the STN recording area, 14 MEcos were between the centre and the upper limit (MEco I lyear), and 14 MEcos were above the upper limit (MEco O l year). The distances between the centre of the STN recording area and its upper limit and the cen- tral point of the MEco I 3 months, MEco O 3 months, MEco I 1 year and MEco O 1 year were calculated (table 2 ). No significant differences were found between the data relative to the MEco I 3 months and the MEco I lyear and between the MEco O 3 months and the MEco O 1 year.

We also compared the data relative to the clinical efficacy of the stimulation on the contralateral wrist rigidity in the four groups (MEco I 3 months, MEco O 3 months, MEco I 1 year, and MEco O lyear), and no significant differences were found $(H=2.562 ; \mathrm{DF}=3 ; \mathrm{p}=0.632)($ table 3$)$.

\section{DISCUSSION}

In this study we found that the mean difference between the position of the STN as identified by the CT-MRI image fusion system using the standard stereotactical coordinates and the central point of the STN area as identified by semimicrorecording were minimal, less than $1 \mathrm{~mm}$ in all the planes. However, in a significant percentage of cases $(35.7 \%)$ it was necessary to modify the stereotactical coordinates to reach the target, always by a medial and posterior correction. The necessity to perform multiple tracks for the reaching of the STN in $35.7 \%$ of cases probably justifies the use of all the strategies (multiple trajectory approach, microstimulation) to correctly reach the target through the functional study of the subthalamic area. ${ }^{413}$

The effects of the stimulation on the different contacts of the electrodes were compared assessing the contralateral wrist rigidity 3 months and 1 year after surgery. The choice to quantify the rigidity improvement rather than the tremor or the akinesia was based on different reasons: the improvement on rigidity due to STN stimulation correlates well with the improvement of all the other parkinsonian symptoms, ${ }^{13}$ it is 
Table 2 Mean values of the distances $(\mathrm{mm})$ between the centre and the upper limit of the STN recording areas and the contacts of the electrodes, grouped in relation to their position inside (MEco I) or above (MEco O) the STN recording area and in relation to the time of follow up ( 3 months and 1 year)

\begin{tabular}{|c|c|c|c|c|c|}
\hline & \multicolumn{2}{|c|}{$\begin{array}{l}\text { Distance from the centre of the STN } \\
\text { recording area }\end{array}$} & \multicolumn{3}{|c|}{$\begin{array}{l}\text { Statistical comparison between } 3 \text { months } \\
\text { and } 1 \text { year data }\end{array}$} \\
\hline & 3 months & 1 year & DF & $F$ & $\mathrm{p}$ Value \\
\hline \multicolumn{6}{|c|}{ MEco I: } \\
\hline$A P$ & $0.5(0.4)$ & $0.5(0.4)$ & 28 & 0.346 & 0.561 \\
\hline Lat & $0.4(0.2)$ & $0.4(0.3)$ & 28 & 0.480 & 0.494 \\
\hline Vert & $1.0(0.6)$ & $1.2(0.6)$ & 28 & 0.519 & 0.477 \\
\hline \multicolumn{6}{|c|}{ MEco O: } \\
\hline $\mathrm{AP}$ & $1.4(0.4)$ & $1.3(0.4)$ & 25 & 0.192 & 0.665 \\
\hline Lat & $1.0(0.4)$ & $0.9(0.4)$ & 25 & 0.049 & 0.826 \\
\hline \multirow[t]{3}{*}{ Vert } & $2.6(0.9)$ & $2.5(0.9)$ & 25 & 0.104 & 0.750 \\
\hline & \multicolumn{2}{|c|}{$\begin{array}{l}\text { Distance from the upper limit of the } \\
\text { STN recording area }\end{array}$} & \multicolumn{3}{|c|}{$\begin{array}{l}\text { Statistical comparison between } 3 \text { months } \\
\text { and } 1 \text { year data }\end{array}$} \\
\hline & 3 months & 1 year & $\mathrm{DF}$ & $F$ & $\mathrm{p}$ Value \\
\hline \multicolumn{6}{|c|}{ MEco I: } \\
\hline$A P$ & $0.5(0.3)$ & $0.4(0.3)$ & 28 & 0.413 & 0.526 \\
\hline Lat & $0.4(0.3)$ & $0.3(0.2)$ & 28 & 1.149 & 0.293 \\
\hline Vert & $1.0(0.5)$ & $0.8(0.5)$ & 28 & 0.601 & 0.445 \\
\hline \multicolumn{6}{|c|}{ MEco O: } \\
\hline$A P$ & $0.4(0.3)$ & $0.3(0.3)$ & 25 & 0.070 & 0.794 \\
\hline Lat & $0.3(0.3)$ & $0.3(0.3)$ & 25 & 0.042 & 0.839 \\
\hline Vert & $1.0(0.9)$ & $0.9(0.8)$ & 25 & 0.061 & 0.807 \\
\hline
\end{tabular}

reproducible, and appears with a short latency. We could not assess the tremor because most of our patients did not show such a symptom, whereas the response of the akinesia to the stimulation has usually a longer latency in respect of rigidity.

Either at 3 months or at 1 year follow up the comparison between the clinical effectiveness of the stimulation on the four different contacts of each electrode always allows us to identify a contact with efficacy higher than the others. The efficacy of the stimulation on the MEcos checked during the contact assessment was confirmed by the UPDRS motor score improvement at 3 months and 1 year.

The mean voltage necessary to obtain the maximal clinical effect on rigidity was almost unchanged at 3 months and at 1 year, suggesting that at 3 months the effect of the STN stimulation is relatively stable not requiring further substantial changes of the parameters of the stimulation to maintain the patients' clinical improvement.

The comparison of the effects of the stimulation on the different contacts at 3 months and 1 year showed that only in three cases ( $11.7 \%$ ) the MEco identified at 1 year was different in respect of the MEco at 3 months, and in all these cases it was above the 3 month MEcos. Comparing the position of the MEco centre with the STN recording area in each patient, only

Table 3 Clinical efficacy of the stimulation on contralateral wrist rigidity (UPDRS item 22)

\begin{tabular}{llll}
\hline & $\begin{array}{l}\text { Basal rigidity } \\
\text { score }\end{array}$ & $\begin{array}{l}\text { Rigidity score } \\
\text { with stimulation }\end{array}$ & Voltage \\
\hline MEco I 3months & $2.5(0.6)$ & $0.3(0.4)$ & $2.7(0.6)$ \\
MEco I ly & $2.5(0.6)$ & $0.1(0.3)$ & $2.9(0.8)$ \\
MEco O 3months & $2.7(0.9)$ & $0.2(0.2)$ & $2.7(0.5)$ \\
MEco O ly & $2.4(0.8)$ & $0.2(0.3)$ & $2.8(0.5)$ \\
\hline
\end{tabular}

Values are means (SD); MEco I 3 months, most effective contacts inside the STN recording area at 3 months; MEco I ly, most effective contacts inside the STN recording area at 1 year; MEco $O 3$ months, most effective contacts above the upper limit of the STN recording area at 3 months; MEco O ly, most effective contacts above the upper limit of the STN recording area at 1 year. in one case at the 3 months follow up was the MEco placed in the lower part of this area, between the centre and the lower limit. At 1 year this finding was not confirmed, because the MEco proved to be in the immediately upper position, or rather, considering the double obliquity of the electrode, more anterodorsolateral. At three months $53.6 \%$ of the MEco centres were inside the STN recording area, between the centre and the upper limit, whereas $42.8 \%$ were outside of the STN recording area, above it. At 1 year, the $50 \%$ of the MEco centres were inside the STN recording area and the 50\% were above it; nevertheless the differences of the position of the contacts between 3 months and 1 year were not significant. At 3 months and at 1 year, the MEcos inside the STN recording area were placed roughly in the middle of its upper part, their centre being at a distance of about $0.5 \mathrm{~mm}$ either from its centre or from its upper limit; moreover, the centres of the MEco outside the STN recording area were at a distance of about 0.5 $\mathrm{mm}$ from its upper limit (table 2).

It has been suggested that the electrical stimulation driven through the surface (roughly $6 \mathrm{~mm}^{2}$ ) of each contact of the definitive quadripolar electrode is capable to affect a tissue area of $2-3 \mathrm{~mm}$ of diameter. ${ }^{8}$ If we consider the trajectory of the electrode, from the anterolateral to the posteromedial, the length of the contacts, and the volume of the tissue area affected by the electrical stimulation it is possible to argue that the effects of the STN stimulation are related to the involvement of the dorsolateral portion of the STN. This finding is consistent with the presence of a functional sharing inside the STN: the dorsolateral portion of the nucleus seems to be involved in the sensorimotor circuits, whereas the ventral portion is connected with associative areas and the medial tip has connections with the limbic system. ${ }^{26}$ It is therefore possible that to obtain a satisfactory clinical improvement of parkinsonian motor symptoms it is important to involve the dorsolateral sensorimotor portion of STN.

The finding that in a significant percentage of cases the MEcos were placed above the STN recording area could be explained by the very short distance (about $0.5 \mathrm{~mm}$ ) between the MEcos and the STN recording area upper limit; it is therefore possible that the clinical effect is related to the spreading 
of the electrical stimulus to the STN. Moreover, the procedure we used to localise the STN (only one recording track in $64.3 \%$ of the cases) could be unable to precisely identify the upper limit of the nucleus in all the cases. Another relevant consideration to explain this finding could be the definition of the MEco simply as the position of its centre. In fact the length of the contact is $1.5 \mathrm{~mm}$, which induces a deviation or plus or minus $0.75 \mathrm{~mm}$ above and below the centre point (a distance which is higher than the $0.5 \mathrm{~mm}$ found for the contacts outside the STN recording area).

Also, during microrecording a displacement of the STN downwards could happen, due to the resistance of the tissue against the electrode providing a deeper recording than actual.

After the macroelectrode positioning a long term adjustment of the brain tissue upwards could explain the finding that 1 year after surgery $50 \%$ of the MEco centres are above the STN as identified by the intraoperative recording area. Finally, an effect due to the involvement of the inhibitory pallidal subthalamic fibres cannot be excluded.

\section{ACKNOWLEDGMENTS}

This study is supported in part by an educational grant from the Compagnia di San Paolo di Torino.

\section{Authors' affiliations}

M M Lanotte, G Faccani, A Melcarne, Department of Neurosurgery, CTO Hospital, Torino, Italy

B Bergamasco, Fondazione Maugeri, University of Torino, Italy

M Rizzone, B Bergamasco, L Lopiano, Department of Neuroscience

\section{REFERENCES}

1 Benabid AL, Benazzouz A, Hoffmann D, et al. Long-term electrical inhibition of deep brain targets in movement disorders. Mov Disord 1998;13:119-25.

2 Krack P, Hamel W, Mehdorn HM, et al. Surgical treatment of Parkinson's disease. Curr Opin Neurol 1999;12:417-25.

3 Starr PA, Vitek JL, Bakay RA. Ablative surgery and deep brain stimulation for Parkinson's disease. Neurosurgery 1998;43:989-1013.

4 Benabid AL, Pollak P, Gao D, et al. Chronic electrical stimulation of the ventralis intermedius nucleus of the thalamus as a treatment of movement disorders. J Neurosurg 1996;84:203-14.

5 Brown RG, Dowsey PL, Brown P, et al. Impact of deep brain stimulation on upper limb akinesia in Parkinson's disease. Ann Neurol 1999:45:473-88.

6 Limousin-Dowsey P, Pollak P, Van Blercom N, et al Thalamic, subthalamic nucleus and internal pallidum stimulation in Parkinson's disease. Neurology 1999;246:S42-5.

7 Kumar R, Lozano AM, Montgomery E, et al. Pallidotomy and deep brain stimulation of the pallidum and subthalamic nucleus in advanced Parkinson's disease. Mov Disord 1998;13:73-82.
8 Pollak P, Benabid AL, Krack P et al. Deep brain stimulation. In: Jankovic $\mathrm{J}$, Tolosa E, eds. Parkinson's disease and movement disorders. Baltimore: Williams and Wilkins, 1998:1085-101.

9 Krack P, Benazzouz A, Pollak P, et al. Treatment of tremor in Parkinson's disease by subthalamic nucleus stimulation. Mov Disord 1998;13:907-14

10 Kumar R, Lozano AM, Sime E, et al. Comparative effects of unilateral and bilateral subthalamic nucleus deep brain stimulation. Neurology 1999;53:561-6.

11 Rodriguez MC, Guridi OJ, Alvarez L, et al. The subthalamic nucleus and tremor in Parkinson's disease. Mov Disord 1998;13:111-18.

12 Yokoyama T, Sugiyama K, Nishizawa S, et al. Subthalamic nucleus stimulation for gait disturbance in Parkinson's disease. Neurosurgery 1999;45:41-7.

13 Limousin $\mathrm{P}$, Krack $\mathrm{P}$, Pollak $\mathrm{P}$, et al. Electrical stimulation of the subthalamic nucleus in advanced Parkinson's disease. N Engl J Med 1998;339:1 105-11

14 Limousin P, Pollak P, Benazzouz A, et al. Effect of parkinsonian signs and symptoms of bilateral subthalamic nucleus stimulation. Lancet 1995;345:91-5.

15 Kumar R, Lozano AM, Kim YJ, et al. Double-blind evaluation of subthalamic nucleus deep brain stimulation in advanced Parkinson's disease. Neurology 1998;51:850-5.

16 Lopiano L, Rizzone M, Bergamasco B, et al. Deep brain stimulation of the subthalamic nucleus: clinical effectiveness and safety. Neurology 2001;56:552-4.

17 Blercom NV, Pollak P, Limousin-Dowsey P, et al. Bilateral subthalamic nucleus stimulation: long term follow-up. Parkinsonism and Related Disorders 1999;5(suppl):S112.

18 Starr PA, Vitek JL, Delong M, et al. Magnetic resonance imaging-based stereotactic localization of the globus pallidus and subthalamic nucleus. Neurosurgery 1999;44:303-13.

19 Walton L, Hampshire A, Forster DM, et al. A phantom study to assess the accuracy of stereotactic localization using $\mathrm{Tl}$-weighted magnetic resonance imaging with the Leksell stereotactic system. Neurosurgery 1996;38:170-6.

20 Benabid AL, Pollak P, Gervason C, et al. Long-term suppression of tremor by chronic stimulation of the ventral intermediate thalamic nucleus. Lancet 1991;337:403-6.

21 Alexander E III, Kooy HM, van Herk M, et al. Magnetic resonance image-directed stereotactic neurosurgery: use of image fusion with computerized tomography to enhance spatial accuracy. J Neurosurg 1995;83:271-6.

22 Hutchison WD, Allan RJ, Opitz H, et al. Neurophysiological identification of the subthalamic nucleus in surgery for Parkinson's disease. Ann Neurol 1998;44:622-8.

23 Bergman $\mathbf{H}$, Wichmann T, Karmon B, et al. The primate subthalamic nucleus. II. Neuronal activity in the MPTP model of parkinsonism. J Neurophysiol 1994;72:507-20.

24 Fahn S, Elton RL, UPDRS Development Committee. The unified Parkinson's disease rating scale. In : Fahn S, Marsden CD, Calne D, ef al, eds. Recent developments in Parkinson's disease. Vol 2. Florham Park, NJ: Macmillan Healthcare Information, 1987:153-63.

25 Schaltenbrand G, Wahren W. Atlas for stereotaxy of the human brain. Stuttgart: Thieme, 1977

26 Parent A. Basal ganglia. In: Parent A, ed. Carpenter's human neuroanatomy. Baltimore: Williams and Wilkins, 1996:838-43. 